\title{
La inoculación de secreción menstrual al cobayo en el diagnóstico de T. B. C. genital femenino
}

\author{
Por Hernando Rodríguez G., M. D. (\%)
}

El presente trabajo tiene por objeto llamar la atención de los médicos generales y de los ginecólogos en especial, sobre un métoco de diagnóstico de T. B. C. genital, inocuo, sencillo y seguro en un alto porcentaje de casos.

La inoculación de secreción menstrual al curí, tal como se ha venido practicando en los últimos diez años en Alemania, Israel, Argentina, Chile, etc., resulta de gran utilidad en el descubrimiento de una entidad entre nosotros muy poco estudiada.

Después de una breve reseña histórica sobre la afección, se presenta el método en cuestión y sus resultados tal como fueron observados por el autor en el Hospital de Clínicas de Buenos Aires, en donde este método de investigación era estudiado por los años de 1956-57 por los doctores Vásquez Ferro (argentino), y Mejía Londoño (colombiano).

Las conclusiones concuerdan en todo con las más ·ecientes obtenidas por investigadores de la autoridad de I. Halbrecht presentadas en reciente artículo del Journal of Obst. \& Gyn.

La historia del estudio de la T. B. C. genital podemos dividirla en varias etapas, así:

Una primera etapa que podríamos llamar del desconocimiento de la entidad, que se extiende hasta los albores del siglo XVIII, en que aparece una de las primeras descripciones, la de Morgagni en la necropsis de una joven de veinte años, que muriera de peritonitis tuberculosa y que presentaba material caseoso en las trompas.

(*) Médico ex-Asistente al Servicio de Ginecología y Esterilidad del Profesor Guillermo Di Paola. Sala 10. Hospital de Clínicas. Buenos Aires. 
Una segunda etapa, en la que la enfermedad era objeto de reconocimiento en exámenes post-mortem, o exámenes histológicos ce piezas obtenidas quirúrgicamente. A ella se hallan vinculados nombres tales como los de Reynaud y Senn (1831), de Louis (1843), äe Virchow (1853) Williams, Hager, Chiari, Koning, Schauta, etc.

Una tercera etapa que abarca desde la segunda o tercera década del presente siglo, hasta mediados de la cuarta. Esta etapa la podriamos denominar etapa de la biopsia endometrial en el diagnóstico pre-operatorio de la T.B.C. genital. Efectivamente, fue este método de examen el que a juiciode los autores latinoamericanos, "permitió modificar fundamentalmente el concepto, pio. fundamente arraigado entre los ginecólogos sobre la dificultax para establecer el diagnóstico de la T. B. C. útero-anexial en el pre-operatorio". Y así se llegó al año de 1952 en que como 10 sostienen los autores chilenos Juan Wood y Humberto Elgueta, mediante la biopsia se pueden diagnosticar en el pre-operatorio por lo menos el $80 \%$ de los casos.

La cuarta etapa o etapa actual, que podemos considerar cronológicamente como que se extiende desde aproximadamente el año de 1950 hasta el momento actual, ha sido una de las más fructíferas, por la serie de métodos nuevos de diagnóstico, tales como los cultivos de sangre menstrual y la inoculación de la misma al cobayo, que han logrado esclarecer un número considerable de casos de T. B. C. genital latentes no identificados por la biopsia endometrial elevando el porcentaje de casos diagnosticables al $90 \%$. como luégo veremos.

A tiempo que se ha avanzado en la manera de investigar, cada vez con mayor exactitud la afección, se han logrado establecer claramente algunas de sus características patogénicas. Por ejemplo:

Se ha establecido que la T. B. C. genital es siempre secundaria a una infección tuberculosa extragenital (pulmonar, ósea, peritoneal, etc.). Que la vía de infección es la hematógena. Que la T. B. C. genital es una entidad mórbida, única en la que la trompa sa compromete en el $100 \%$ de los casos, y en la que la bilateralidad de la lesión prácticamente alcanza el mismo porcentaje.

El compromiso del endometrio se comprueba en el $85 \%$ de los casos; el de los ovarios, en el $25 \%$, y el del cuello en aproximadamente el 15 al $17 \%$ de los casos.

Métodos de examen.-Decíamos cómo la biopsia endometrial fue el primero en ser empleado con buenos resultados, tan buenos 
que aún hoy, a pesar de los exámenes de reciente invención no ha podido ser desplazada y conserva todo su valor. Es el examen de elección en investigación de T. B. C. endometrial. No sirve, sinembargo, para el diagnóstico de los casos de T. B. C. anexial incipiente y da resultados falsamente negativos en un $20 \%$ de los casos, aproximadamente. Otro método de examen es la histerosalpingografía, que no ha contado con la suficiente acogida por parte de la mayoría de los ginecólogos latinoamericanos que se muestran reservados de su valor (quizá por falta de confianza en los radiólogos), al paso que los europeos y los judíos, como lo veremos más adelante, le conceden un valor que casi supera al de la biopsia por su fidelidad. Es de incuestionable valor en el diagnóstico de T. B. C. anexial.

Los cultivos de sangre menstrual y de secreción intermenstrual para identificación de micobacterium tuberculoso también han sido empleados. $\mathrm{Y}$ los cultivos de secreciones servical y vaginal como método de investigación en casos de amenorrea $y$ de enfermas vírgenes.

Finalmente, la inoculación de la sangre menstrual en el curi que es el método del que me ocuparé a continuación.

En el año de 1954 uno de los médicos del Servicio de Ginecoogía del Hospital Rivadavia viajó a Alemania con el fin de adelantar estudios de especialización en algunos centros de ese país. $Y$ allí pudo observar cómo los investigadores alemanes empleaban sn vía de ensayo "la inoculación de la sangre menstrual al cobayo" como método para descubrir los casos de T. B. C. genital latente; y a su regreso a Buenos Aires introdujo el método.

Así es como ha venido practicándose desde el año de 1955, primero en el Hospital Rivadavia y luégo en el Hospital de Clínicas, donde tuve ocasión de conocerlo de cerca como médico asistente en el Servicio del profesor Di Paola, durante el año de 1957.

Las observaciones se hicieron sobre un total de 74 pacientes entre todas las que consultaron por esterilidad primaria, y sus re sultados fueron publicados por primera vez en agosto del año pasado en unas Jornadas Ginecológicas realizadas en Montevideo.

El procedimiento es extraordinariamente sencillo. No molesta a la enferma. No tiene ninguno de los riesgos que puede encerrar la biopsia en los casos de infección genital crónica. Su fidelidad, comparada con la de la biopsia es mayor. Y, en fin, tiene indicaciones más amplias que ellas, como son la de detectar los casos de T. B. C. genital latente y anexial incipiente. 
Cómo se hace el examen.-Se toma la muestra de la descarga menstrual el primer día del ciclo, para lo cual se instruye a la $乞 n-$ ferma a fin de que concurra a su médico en esa fecha.

El médico coloca una "cápsula" en el cuello, que recoge las secreciones uterinas que fluyen por el orificio exterior del cervix.

Casi siempre en el mismo momento del examen se obtiene la cantidad deseada. Ocasionalmente hay que dejar la cápsula por algunas horas antes de retirarla.

La muestra así obtenida se macera. Luégo se trata con solución salina en proporción del 10\% y se centrifuga a 3.500 revoluciones por cinco minutos. De la parte superior del centrifugado se toman 5 c. c que se inyectan con una aguja y jeringa hipodérmicas en la cara interna del muslo del cobayo (entre nosotros, en el Hospital San José, se acostumbra a inyectar 1 c. c. nada más).

Desda este instante comienza la observación, que, por lo demás, no quita mucho tiempo, pues se reduce a observar las manifestaciones mórbidas que tenga el animal, sus características, ete,. a fin de poder prever si su muerte ocurrirá en poco tiempo.

Puede ocurrir que el animal muera antes de 45 días. Se le practica autopsia y sus ganglios y vísceras son enviadas al laboratorio de anatomía patológica a fin de investigar lesiones T. B. C.

Si el animal sobrevive a los 45 días de la inoculación, se sacrifica $\mathrm{y}$ se procede como en el caso anterior.

¿Qué encontramos macroscópicamente en estos animales sacrificados? Lo más frecuente es la hipertrofia de los ganglios hipogástricos, que alcanzan casi siempre el tamaño de un garbanzo, mientras que en los casos negativos no se hallan adenopatías. Los ganglios se observan como granos de mijo.

Lesiones a su vez en el peritoneo, con aspecto de pequeños nódulos blanquecinos, lo mismo sobre los puimones, hígado, bazo, etc.

Resultados.-El total de enfermos se repartió en tres grupos: 35 casos (o sea el 50\%) del total de 54 casos estudiados fueron controlados por biopsia e inoculación.

Nueve casos (10\%) fueron controlados con cultivo de la descarga menstrual e inoculación en el medio de Petragnani y el resto, o sean $60 \%$, por inoculación solamente.

En el primer grupo fueron positivos 34 a la biopsia y un caso que en una primera biopsia practicada en 1955, dio evidencia de lesión T. B. C.; cuatro biopsias posteriores practicadas por otros anatomapatólogos fueron negativas. La inoculación fue positiva, 
y una quinta biopsia hecha después de obtenido el resultado de la inoculación fue positiva. Se trataba de una T. B. C. anexial constatada por histerosalpingografía.

Todos los casos positivos en el segundo grupo, lo fueron a la inoculación también.

$\mathrm{Y}$ hubo cuatro casos del tercer grupo con resultado negativo que fueron intervenidos por pensarse en otras entidades que posteriormente al estudio histológico de la pieza dieron T. B.C.

Halbrecht, sobre 120 casos estudiados en los últimos 10 años, empleó como métodos de diagnóstico: la biopsia endometrial, el cultivo de secreciones uterinas y la inoculación de secreción menstrual al curí en forma simultánea, y observó lo siguiente:

a) En un primer grupo de 54 pacientes la biopsia y los cultivos fueron positivos ambos.

b) En 38 enfermas de un segundo grupo la inoculación fue positiva, mientras la B. mostraba endometrio normal.

c) En un tercer grupo de 11 pacientes la biopsia dio resultados positivos, y los cultivos e inoculación negativas.

d) En un cuarto grupo de 17 enfermas, solo se usuó el cultivo: todas con resultados positivos.

Halbrecht llegó a la conclusión que mientras la biopsia endometrial fue positiva en 65 casos: o sea el $63 \%$ de sus pacientes, la inoculación lo fue en 92 , o sea el $90 \%$.

\section{COMENTARIO}

La T. B. C. genital, afección poco estudiada entre nosotros, es, no obstante, una causa importante de esterilidad. Durante más de 20 años el único método de diagnóstico seguro para detectarla fue la biopsia endometrial.

Pero muchos casos de T. B. C. clínicamente asintomáticos pasarían desapercibidos si nos atuviésemos solamente al método-diagnóstico de la biopsia.

La inoculación de secreción menstrual al curí ha ayudado a muchos investigadores en los últimos 10 años a descubrir un número de casos de T. B.C. genital latente que la biopsia no pudo revelar y en algunos casos como la T. B. C. anexial incipiente fue el único método capaz de diagnosticarla.

Esto encierra una gran importancia, pues como anota Halbrecht "es evidente la necesidad de descubrir la T. B. C. anexial en su estado incipiente. No obstante que las lesiones tuberculosas 
de los órganos genitales femeninos generalmente muestran tendencia hacia la autolimitación, el resultado, si este proceso no se detiene antes de la etapa endometrial, es siempre la esterilidad.

Este solo hecho hace a nuestro juicio aconsejable el método, en atención a que: primero: es fácil de realizar; segundo: no encierra ningún peligro para la salud o la vida de la paciente; tercero: no representa ninguna molestia para ella; cuarto: se muestra tan fiel como la biopsia endometrial para descubrir los casos de T. B. C. endometrial, y es superior a ella en la investigación de T. B. C. genital latente y anexial.

\section{REFERENCIAS}

1. ARIGHI LEONCIO y SARDI J. L.-“"Esterilidad por salpingitis T. B. C. Latente; conducta del ginecólogo frente a estas sospechas". Obst y Gin Latino-Am. Vol. IX. Página 51, 4:48.

2. CHEVALIER RAUL y SANMARTINO R.-"Permeabilidad tubaria y T. B. C.” Obst. y Gin. Lat. Am. Vol. IX. 1951. Página 450.

3. FERREYRA DOS SANTOS.- "Tuberculose do miometrio". Obs. y Gin. Lat. Am. Vol. X. 1952. Página 214.

4 WOOD JUAN ELGUETA H.- "Conceptos modernos sobre tuberculosis útero-anexial". Obst. y Gin. La Am. Vol. X. Página 254.

5. HALBRECHT' I. M. D. ISRAEL.-“Valor relativo del cultivo y la E. E. en el çiagnóstico de la T. B. C. genital". A. J. of Obs. \& Gyn. 1858. Vol. LXXV. Página 899. 